\title{
Language identification from visual-only speech signals
}

\author{
Rebecca E. Ronouest \\ Indiana University, Bloomington, Indiana \\ SuSANNAH V. LeVI \\ New York University, New York, New York \\ AND \\ DaVId B. Pisoni \\ Indiana University, Bloomington, Indiana
}

\begin{abstract}
Our goal in the present study was to examine how observers identify English and Spanish from visual-only displays of speech. First, we replicated the recent findings of Soto-Faraco et al. (2007) with Spanish and English bilingual and monolingual observers using different languages and a different experimental paradigm (identification). We found that prior linguistic experience affected response bias but not sensitivity (Experiment 1). In two additional experiments, we investigated the visual cues that observers use to complete the languageidentification task. The results of Experiment 2 indicate that some lexical information is available in the visual signal but that it is limited. Acoustic analyses confirmed that our Spanish and English stimuli differed acoustically with respect to linguistic rhythmic categories. In Experiment 3, we tested whether this rhythmic difference could be used by observers to identify the language when the visual stimuli is temporally reversed, thereby eliminating lexical information but retaining rhythmic differences. The participants performed above chance even in the backward condition, suggesting that the rhythmic differences between the two languages may aid language identification in visual-only speech signals. The results of Experiments $3 \mathrm{~A}$ and $3 \mathrm{~B}$ also confirm previous findings that increased stimulus length facilitates language identification. Taken together, the results of these three experiments replicate earlier findings and also show that prior linguistic experience, lexical information, rhythmic structure, and utterance length influence visual-only language identification.
\end{abstract}

A large body of research has demonstrated that speech perception is multimodal in nature. In addition to the auditory properties of speech, the visual signal carries important information about the phonetic structure of the message that affects the perception and comprehension of the speech signal (see, e.g., Massaro, 1987; Sumby \& Pollack, 1954; Summerfield, 1987). The visual aspects of speech have been shown to both enhance and alter the perception of the auditory speech signal for listeners with hearing impairment, as well as for normal-hearing listeners (see, e.g., R. Campbell \& Dodd, 1980; Hamilton, Shenton, \& Coslett, 2006; Kaiser, Kirk, Lachs, \& Pisoni, 2003; Lachs, 1999; Lachs, Weiss, \& Pisoni, 2002; Summerfield, 1987).

In their seminal study of audio-visual speech perception, Sumby and Pollack (1954) showed that the addition of visual information dramatically improved speech intelligibility at less favorable signal-to-noise ratios in normalhearing listeners. When presented with degraded auditory signals, the observers experienced large gains in intelligibility of speech signals in the auditory-visual conditions relative to the auditory-only conditions. The contribution of visual information to speech perception is also illustrated by the McGurk effect (McGurk \& MacDonald, 1976), in which conflicting auditory and visual information alters perception. McGurk and MacDonald found that when observers were presented with mismatched auditory and visual information, they perceived a sound that was not present in either sensory modality. For example, a visual velar stop (/g/) paired with an auditory bilabial stop (/b/) was perceived as $/ \mathrm{d} /$. Thus, the information carried by the visual signal not only enhances speech perception, as was found by Sumby and Pollack, but can also alter the perception of auditory information, yielding a novel percept, as in the McGurk effect (see also Hamilton et al., 2006).

More recently, studies in the field of second language (L2) acquisition have shown that the addition of visual information aids in the acquisition and perception of nonnative contrasts. For example, Hardison (2003) examined the acquisition of the English $/ 1 /-/ r /$ contrast by native Japanese and Korean speakers who were trained using either auditory-only or auditory-visual signals. The learners who were trained in the auditory-visual condition showed

R. E. Ronquest, rronques@indiana.edu 
better identification of $/ \mathrm{l} /$ and $/ \mathrm{r} /$ in the posttest than those participants who were trained in auditory-only conditions. Similarly, Hazan, Sennema, and Faulkner (2002) found that visual information is available to L2 learners, but only once the auditory contrast has been acquired. Spanish learners of English who perceived the English /b/-/v/ contrast in the auditory-only condition also perceived the difference in the visual-only condition. In contrast, learners at early stages of acquisition who demonstrated low rates of auditory identification of $/ \mathrm{b} /$ and $/ \mathrm{v} / \mathrm{did}$ not benefit from the addition of the visual presentation. Navarra and Soto-Faraco (2007) reported similar findings for the perception of the Catalan /e/-/e/ contrast by Spanish and Catalan bilinguals. Spanish-dominant bilinguals did not distinguish between the phonemes in the auditory-only condition but were sensitive to the contrast when the visual correlates were included. Taken together, the results of these studies demonstrate that visual cues enhance the discrimination and perception of L2 targets in difficult phonetic environments and are an additional source of phonetic information for L2 learners.

In a related study, Werker, Frost, and McGurk (1992) reported that the proportion of visual-capture (i.e., when the visual signal overrides the auditory signal) responses in a McGurk-type task was affected by the participants' native language and previous L2 experience. L1 and L2 speakers of French and English were presented with a multimodal stimulus that consisted of conflicting auditory and visual information; auditory /ba/ was paired with visual /ba/, /va/,/ða/,/da/,/3a/, and /ga/. Werker et al. found that beginning and intermediate $\mathrm{L} 2$ learners of English demonstrated significantly less visual capture of the interdental place of articulation of /ð/ than did more proficient speakers of English. The beginning and intermediate learners of English generally reported hearing /ta/ or /da/; thus, they had assimilated the interdental place of articulation with that of the closest French phoneme (/t/ or $/ \mathrm{d} /)$. In contrast, native English speakers, true bilinguals, and advanced English learners were more influenced by the visual stimulus and demonstrated a higher percentage of /ða/ responses. Werker et al. concluded that the ability to incorporate visual information during speech perception is highly dependent on experience with that language.

Taken together, these studies show that the visual information encoded in a multimodal speech signal contributes substantially to speech intelligibility and that past linguistic experience and developmental history affect how and to what extent visual information is processed. Although previous research on visual speech perception and speechreading has been focused primarily on examining participants' ability to identify specific segments or words in a particular language, whether languages can be discriminated or identified on the basis of only visual information has not been directly examined until recently.

In two recent studies, visual-only language discrimination was investigated in adult and infant observers. SotoFaraco et al. (2007) examined whether monolingual and bilingual observers could discriminate Spanish from Catalan with visual-only speech stimuli. Two groups of bilinguals (Spanish dominant, Catalan dominant) and three groups of monolinguals (Spanish, Italian, English) completed the task. The bilingual participants discriminated the languages better than the monolingual Spanish participants, who still performed above chance. The English and Italian monolingual observers, who were familiar with neither language, were not successful at the task, suggesting that knowledge of at least one of the languages is necessary for visual-only discrimination. Soto-Faraco et al. concluded that prior experience with the specific languages is one of the primary factors contributing to successful discrimination. They suggested that a number of different aspects of the stimuli facilitated discrimination, such as the length of the utterance and the number of distinctive segments or words present in the stimulus. A similar study conducted with infants showed that 4 to 6-month-olds can discriminate between French and English in visual-only displays but that by 8 months of age, this ability is limited only to bilingual infants (Weikum et al., 2007).

Soto-Faraco et al. (2007) suggested that future investigations should examine observers' ability to discriminate or identify languages that are less closely related than Spanish and Catalan. In the present study, we sought to replicate Soto-Faraco et al.'s findings with English and Spanish, two languages that differ in rhythmic classification and timing (e.g., Grabe \& Low, 2002; Pike, 1946; Ramus \& Mehler, 1999; Ramus, Nespor, \& Mehler, 1999), in order to examine the contribution of rhythmic information in visual-only language processing. Spanish is a syllable-timed language, whereas English is a stresstimed language. Syllable-timed languages exhibit more even spacing of syllables in an utterance (Pike, 1946), measured by variability of vowel durations. Thus, the duration of vowels in an utterance is more regular in syllabletimed languages (Grabe \& Low, 2002). In contrast, vowel durations in stress-timed languages are highly variable. For example, English exhibits extensive vowel reduction and shortened duration of unstressed vowels.

A considerable body of literature demonstrates that rhythmic information is salient in the auditory domain and that listeners can distinguish between languages in the absence of lexical or segmental information by relying solely on linguistic rhythm and durational cues. In one study, Ramus and Mehler (1999) resynthesized English and Japanese utterances by replacing all consonants with /s/ and all vowels with /a/ and overlaying a flat $f 0$ contour on these segmentally simplified utterances. This manipulation thus eliminated lexical, segmental, and pitch differences across the languages but retained the relative duration of consonants and vowels (i.e., rhythmic differences). French listeners were able to discriminate these English and Japanese utterances with these rhythmic cues alone. Crucially, English and Japanese belong to different rhythmic categories (stress-timed and syllable-timed, respectively). Numerous studies have also shown that even prelinguistic infants can discriminate languages on the basis of their rhythmic properties (Mehler et al., 1988; Nazzi, Bertoncini, \& Mehler, 1998; Nazzi, Jusczyk, \& Johnson, 2000), suggesting that 
sensitivity to linguistic rhythm and temporal properties of speech develops early and is a highly salient property that differentiates languages of the world.

In previous studies of linguistic rhythm, the acoustic (i.e., auditory) properties of the signal, measured by variability of the duration of consecutive vowels, have been examined (e.g., Grabe \& Low, 2002). Thus, in terms of the acoustics, stress-timed languages such as English exhibit a large amount of variation in vowel duration from one vowel to another, whereas syllable-timed languages such as Spanish show much less variability of vowel duration. These rhythmic, durational differences that have been found in the acoustic domain have parallel correlates in the visual domain, because these two sensory modalities are closely coupled.

Work on visual-only speech perception demonstrates that consonants and vowels map onto distinct visemes (e.g., C. S. Campbell \& Massaro, 1997; Montgomery \& Jackson, 1983; Rosenblum \& Saldaña, 1998; Walden, Prosek, Montgomery, Scherr, \& Jones, 1977). Thus, observers of visual speech can distinguish whether a particular viseme corresponds to the segmental class of vowels or to that of consonants. Furthermore, the duration of the vocalic opening (i.e., the vowel duration) during the production of vowel visemes is also present in a visual display. In fact, duration is used in C. S. Campbell and Massaro as a feature that differentiates similar visemes, suggesting that duration is indeed perceptible in visual speech signals. Taken together, the research on visual-only speech perception suggests that the duration of consonant and vowel visemes is available to observers. Thus, the combined evidence from auditoryrhythmic studies showing that the durational variability of vowels can indicate rhythmic class and from viseme categories, which carry visual information specifying phonetic distinctions (consonant vs. vowel) and duration, suggests that observers have access to rhythmic properties in the visual signal specified by the durational variability of consecutive vocalic visemes. Furthermore, observers should be able to use these differences in vocalic viseme duration variability (i.e., rhythmic differences) to differentiate languages that belong to different rhythmic categories. Translating acoustic into visual correlates, the vocalic visemes in Spanish will be more regular in duration, whereas the vocalic visemes in English will show more variability with respect to duration. Therefore, another of our goals in the present investigation was to test whether observers can use rhythm-class differences to aid language identification from visual-only displays of speech.

Our first goal was to replicate the findings of SotoFaraco et al. (2007). In Experiment 1, we tested language identification with monolingual and bilingual SpanishEnglish participants using a two-alternative forced choice (2AFC) task. Soto-Faraco et al. convincingly showed that observers used both lexical and segmental cues to differentiate languages. Our second goal in the present investigation was to examine additional cues available to observers for language identification. In Experiments 2 and 3 , we manipulated several aspects of the visual signal to examine the availability of lexical information and rhythmic cues in visually presented displays of speech. In Experiment 2, we tested observers' ability to identify lexical information. In Experiment 3, we examined acoustic differences between Spanish and English to confirm that the stimuli used here exhibit differences in rhythmic structure. In Experiment 3, we also investigated the role of this rhythmic difference in visual-only language identification.

\section{EXPERIMENT 1 Visual-Only Language Identification}

\section{Method}

Stimulus materials. The stimulus materials used in Experiment 1 consisted of a set of visual-only video clips of 20 English and 20 Spanish sentences spoken by a male and a female bilingual speaker (see Appendix A). The recordings were made using a Behringer B1 Studio Condenser microphone and a Panasonic AG-DVX100 video recorder. All of the recordings were made in a sound-attenuated IAC booth in the Speech Research Laboratory at Indiana University. The male talker was a native of Venezuela, and the female talker was a native of Puerto Rico. Both talkers acquired English during early adolescence and had lived in the United States for at least 6 years at the time of the recording.

Participants. Four groups of participants were recruited for Experiment 1: monolingual English speakers $(n=16)$, monolingual Spanish speakers $(n=12)$, English-dominant bilinguals $(n=16)$, and Spanish-dominant bilinguals $(n=12)$. The monolingual English participants were all undergraduate students at Indiana University who reported little or no knowledge of Spanish. The monolingual Spanish observers were all residents of Caracas, Venezuela, who reported that they did not speak or have knowledge of English. The Spanish-dominant bilinguals and the English-dominant bilinguals were all graduate students at Indiana University who reported that they were proficient speakers of both Spanish and English. They all had some experience teaching college-level Spanish (Table 1). The ages of L2 acquisition for these bilinguals ranged from birth to 19 years of age. None of the participants reported a history of speech or hearing disorders at the time of testing. All of the participants received $\$ 10$ for taking part in the study.

Procedure. The stimuli were presented to the bilingual and monolingual English-speaking participants on an Apple Macintosh G4 computer. The monolingual Spanish speakers completed the experiment on an Apple Macintosh iBook G3 notebook computer in Caracas, Venezuela. PsyScript Version 5.1 was used for stimulus presentation. The participants' responses were recorded with a button box for the language-identification task. The entire experiment took approximately $1 \mathrm{~h}$ to complete.

The visual-only language-identification task consisted of two blocks of 40 video clips of short meaningful sentences in Spanish and English (see Appendix A). The stimuli were blocked by talker gender and counterbalanced across participants. After seeing each video clip, the participants were asked to decide whether the person in the video was speaking English or Spanish. No feedback was provided.

Data analysis. In a $2 \mathrm{AFC}$ task, percentage correct scores are influenced by both sensitivity and bias. For this reason, nonparametric measures of sensitivity $\left(A^{\prime}\right)$ and bias $\left(B_{\mathrm{D}}^{\prime \prime}\right)$, which use both hit and false alarm rates, were calculated for each participant to obtain robust measures of performance (Donaldson, 1992; Grier, 1971). In Experiments 1,3A, and 3B, a response of English to English stimuli was considered a hit; a response of English to Spanish stimuli was considered a false alarm.

Sensitivity $\left(A^{\prime}\right)$ was measured on a scale of $0-1$, with .5 indicating chance performance and 1 indicating perfect discrimination. Donaldson's (1992) bias ( $\left.B_{\mathrm{D}}^{\prime \prime}\right)$ was also used. It is measured on a scale of -1.0 to 1.0. A score of 0 indicates no response bias. In Ex- 
Table 1

Demographic Information Pertaining to the Bilingual Participants in Experiment 1

\begin{tabular}{|c|c|c|c|c|}
\hline Participant & Age & Age L2A & L2 Fluency & Country \\
\hline \multicolumn{5}{|c|}{ English-Dominant Bilinguals } \\
\hline 1 & 28 & 19 & Good & USA \\
\hline 2 & 24 & 14 & Good & USA \\
\hline 3 & 23 & 17 & Good & USA \\
\hline 4 & 22 & 13 & Good & USA \\
\hline 5 & 24 & 14 & Good & USA \\
\hline 6 & 23 & 15 & Good & USA \\
\hline 7 & 30 & 0 & Good & USA \\
\hline 8 & 25 & 12 & Good & USA \\
\hline 9 & 25 & 17 & Good & USA \\
\hline 10 & 31 & 19 & Good & USA \\
\hline 11 & 26 & 12 & Good & USA \\
\hline 12 & 35 & 15 & Good & USA \\
\hline 13 & 27 & 14 & Good & USA \\
\hline 14 & 26 & 14 & Good & USA \\
\hline 15 & 33 & 15 & Good & USA \\
\hline 16 & 23 & 14 & Good & USA \\
\hline \multicolumn{5}{|c|}{ Spanish-Dominant Bilinguals } \\
\hline 1 & 25 & 11 & Good & Mexico \\
\hline 2 & 25 & 15 & Good & Colombia \\
\hline 3 & 26 & 6 & Good & Mexico \\
\hline 4 & 28 & 14 & Good & Spain \\
\hline 5 & 24 & 13 & Good & Colombia \\
\hline 6 & 31 & 18 & Good & Colombia \\
\hline 7 & 36 & 9 & Good & Uruguay \\
\hline 8 & 39 & 17 & Good & Colombia \\
\hline 9 & 26 & 0 & Good & USA \\
\hline 10 & 26 & 12 & Good & Spain \\
\hline 11 & 26 & 10 & Good & Spain \\
\hline 12 & 25 & 15 & Good & Spain \\
\hline
\end{tabular}

Note-Second language (L2) fluency was rated on a scale of poor-fairgood. The 2 bilinguals who learned their L2 at birth were grouped with the English-dominant or Spanish-dominant groups on the basis of which language was used more on a daily basis. Age L2A, age of L2 acquisition (in years).

periments $1,3 \mathrm{~A}$, and $3 \mathrm{~B}$, negative bias scores denote a tendency to respond English when presented with a stimulus, and positive values indicate a tendency to respond Spanish.

\section{Results}

To determine whether the participants' sensitivity was above chance performance (above .5 on the sensitivity scale), one-sample $t$ tests were conducted for each group of participants. As is shown in Figure 1, sensitivity for all four participant groups was significantly above chance [monolingual English, $t(15)=17.72, p<.001$; Englishdominant bilinguals, $t(15)=28.30, p<.001$; monolingual Spanish, $t(11)=20.93, p<.001$; Spanish-dominant bilinguals, $t(11)=9.03, p<.001]$. A one-way ANOVA was conducted on the $A^{\prime}$ scores with participant group as a between-subjects factor. The results of this analysis were not significant, suggesting that all four groups performed comparably and that the observers' ability to identify a stimulus as Spanish or English did not depend on their native language or prior language experience.

The mean bias $\left(B_{\mathrm{D}}^{\prime \prime}\right)$ scores for all four participant groups are shown in Figure 2. A one-sample $t$ test of $B_{\mathrm{D}}^{\prime \prime}$ scores showed that only the English-dominant bilinguals showed a response bias that differed significantly from 0 $[t(15)=-4.18, p<.001]$; the English-dominant bilin- guals had a strong tendency to choose the English response option, whereas the other three groups of participants did not demonstrate a significant bias for either response alternative. A one-way ANOVA on the $B_{\mathrm{D}}^{\prime \prime}$ scores revealed a significant difference between the groups $[F(3,52)=$ 5.08, $p=.004]$. Post hoc Tukey tests revealed that the English-dominant bilinguals displayed a response bias that was significantly different from that of the English monolinguals ( $p=.03)$ and from that of the Spanishdominant bilinguals $(p=.003)$. Although both groups of bilingual participants showed a tendency to respond with their native language, the bias was strongest for the group of English-dominant bilinguals.

\section{Discussion}

The results of Experiment 1 provide additional insights into the robustness of language identification from visualonly displays of speech. Several findings first reported in Soto-Faraco et al. (2007) were replicated in the present

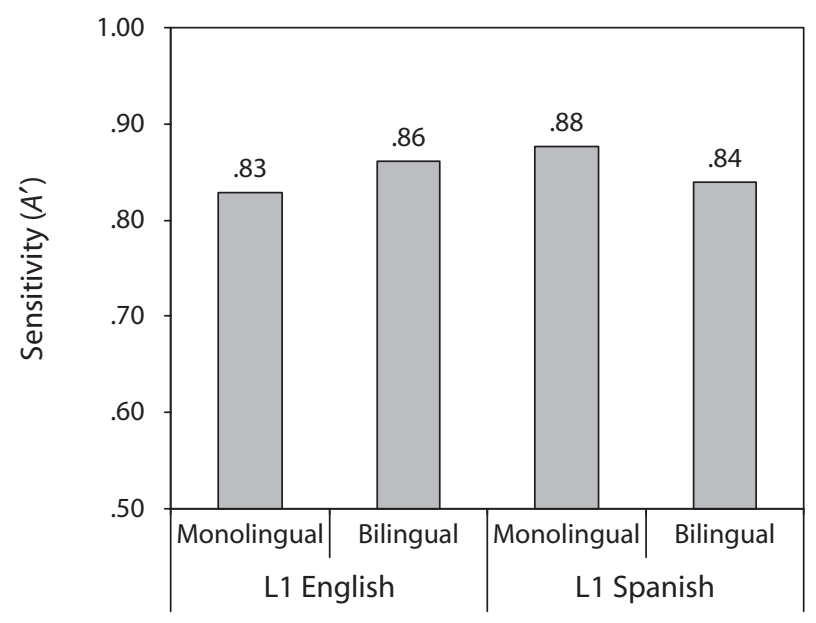

Figure 1. Mean sensitivity $\left(A^{\prime}\right)$ for all four participant groups for Experiment 1.

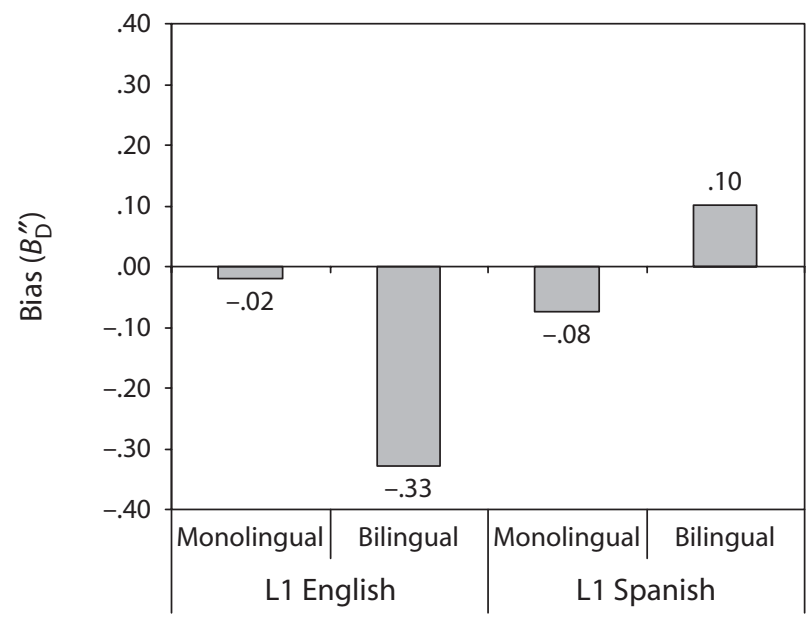

Figure 2. Mean bias ( $\left.\boldsymbol{B}_{\mathrm{1}}^{\prime \prime}\right)$ for all four participant groups in $\mathbf{E x}$ periment 1. Negative values indicate a bias to respond English; positive values indicate a bias to respond Spanish. 
study. First, regardless of language background or prior linguistic experience, all of the participants performed significantly above chance. This result suggests that the visual signal alone provides sufficient information for an observer to correctly identify the language being spoken. The finding that both the monolingual and the bilingual observers completed this task successfully replicates the earlier results of Soto-Faraco et al., who found that knowledge of one of the test languages was sufficient to allow visual-only discrimination of Spanish and Catalan. The present results show that using different languages and a different experimental paradigm yields findings similar to those reported by Soto-Faraco et al.

Unlike Soto-Faraco et al. (2007), however, we did not find significant differences in sensitivity $\left(A^{\prime}\right)$ between the four participant groups. The monolingual participants were as sensitive as the bilingual participants at identifying which language was spoken in the video clips. It may be that the monolingual participants performed the 2AFC task by deciding whether the stimulus was presented in their L1 or not in their L1, rather than making an English versus Spanish judgment.

Measures of response bias $\left(B_{D}^{\prime \prime}\right)$ revealed that the two bilingual participant groups exhibited some preference to respond with their native language. The response bias was particularly strong in the group of English-dominant bilinguals. A trend was observed for the bilinguals to show more response bias than the monolinguals, although this difference failed to reach statistical significance for the native Spanish-speaking participants. The difference in performance between monolingual and bilingual observers may be due to the perceived familiarity of the visual utterances. Monolingual English and Spanish speakers who possessed knowledge of only one of the two test languages may have relied on familiarity of a particular utterance (e.g., recognizing a familiar word or temporal pattern in their L1). When no familiar words or patterns were present, making the stimulus appear unfamiliar, they may have decided that the utterance was in the nonnative (unfamiliar) language. In the case of the bilinguals, all of the video clips had the potential to contain familiar words, segments, syllable structures, or rhythmic information in either language; thus, all utterances would have appeared familiar. On finding some degree of familiarity in the signal, the bilinguals may have processed the visual signal as belonging to the $\mathrm{L} 1$ because of $\mathrm{L} 1$ dominance.

The lack of a statistically significant response bias toward Spanish for the Spanish bilinguals may be the result of the way in which the task was administered to this group. All paperwork and instructions were presented to the Spanish-dominant bilinguals in their nonnative language (English), whereas the English-dominant bilinguals received paperwork and task instructions in their native language. Thus, using their nonnative language as the primary mode of presentation may have attenuated the native language bias by putting the Spanish-dominant bilinguals in an English mode.

Although we replicated the basic findings reported by Soto-Faraco et al. (2007), neither their study nor Experiment 1 was designed to explain how the participants car- ried out the visual-only language-identification task. What visual cues do observers use to identify the language spoken in visual-only speech? In the remaining experiments in this article, we attempted to examine the contribution of lexical information, stimulus length, and rhythmic information on visual-only language identification. Unlike in Experiment 1, which was designed to examine differences in language identification due to linguistic experience, in Experiments 2 and 3, we tested the contribution of different types of information available in the visual signal. Only monolingual English speakers took part in the remaining experiments. Monolingual English speakers were chosen for two reasons. First, the results of Experiment 1, as well as those of Soto-Faraco et al., suggest that knowledge of one language is sufficient for visual-only language identification and discrimination tasks. Second, the monolingual English speakers in Experiment 1 did not perform differently from the bilingual participants and showed less response bias.

\section{EXPERIMENT 2 Availability of Lexical Information}

Previous research on lipreading has shown that lexical and segmental information can be extracted from visualonly speech (Bernstein, Demorest, \& Tucker, 1998; Demorest \& Bernstein, 1992; Kaiser et al., 2003; Lachs, 1999; Lachs et al., 2002). In Experiment 2, we examined the contribution of lexical information to visual-only language identification in order to determine the extent to which the observers in Experiment 1 may have used lexical knowledge to carry out the language-identification task.

\section{Method}

Stimulus materials. The stimulus materials used in Experiment 2 consisted of the same English and Spanish sentences used in Experiment 1.

Participants. Ten monolingual English speakers who reported no history of speech or hearing disorders participated in Experiment 2. The participants reported that they had little or no knowledge of an L2, and none had received formal instruction in Spanish. All of the participants were paid $\$ 7$ for taking part in the experiment. None of the participants in Experiment 2 had completed Experiment 1.

Procedure. The participants were presented with a single block of 80 trials mixed by talker and stimulus language. The participants were instructed to watch the video clip and type what they thought the person said. The participants were not informed whether the video clips were spoken in English or Spanish. No feedback was provided.

\section{Results}

The participants' responses were scored according to the total number of words identified correctly on each trial. Examination of the participants' responses indicated that for the English stimuli only, the participants averaged 26\% total words correct, although this varied greatly for the different sentences. The mean number of words correct and the standard deviation for each of the English sentences is represented in Table 2. Because all of the participants in this experiment were monolingual English speakers, they were unable to correctly identify any words in the Spanish sentences. The responses to these stimuli consisted of English words, nonsense words, or no response. 
Table 2

Mean Percentage of Words Correct and Standard Deviations for English Sentences Spoken by Both Talkers

\begin{tabular}{|c|c|c|c|c|}
\hline \multirow[b]{2}{*}{ English Sentence } & \multicolumn{2}{|c|}{$\begin{array}{c}\text { Talker } 1 \\
\text { (Male) }\end{array}$} & \multicolumn{2}{|c|}{$\begin{array}{l}\text { Talker } 2 \\
\text { (Female) }\end{array}$} \\
\hline & $M(\%)$ & $\overline{S D}$ & $M(\%)$ & $S D$ \\
\hline Wait just a minute! & 0 & 0 & 20 & 42 \\
\hline Breakfast is ready. & 50 & 48 & 57 & 50 \\
\hline I don't know what's wrong with the car, but it won't start. & 21 & 24 & 57 & 36 \\
\hline It sure takes a sharp knife to cut this meat. & 10 & 28 & 4 & 7 \\
\hline I haven't read a newspaper since we bought a television set. & 12 & 15 & 15 & 10 \\
\hline Weeds are spoiling the yard. & 8 & 14 & 2 & 6 \\
\hline Call me a little later. & 16 & 26 & 22 & 29 \\
\hline Do you have change for a five-dollar bill? & 49 & 46 & 34 & 39 \\
\hline How are you? & 50 & 53 & 57 & 45 \\
\hline I'd like some ice cream with my pie. & 23 & 26 & 25 & 26 \\
\hline Where can I find a place to park? & 24 & 28 & 39 & 39 \\
\hline I don't think I'll have any dessert. & 16 & 21 & 36 & 25 \\
\hline I like those big red apples we always get in the fall. & 37 & 27 & 18 & 7 \\
\hline The show's over. & 17 & 32 & 16 & 25 \\
\hline You'll get fat eating candy. & 30 & 33 & 50 & 39 \\
\hline Why don't they paint their walls some other color? & 2 & 5 & 6 & 9 \\
\hline What's new? & 45 & 28 & 30 & 42 \\
\hline What are you hiding under your coat? & 13 & 14 & 24 & 15 \\
\hline How come I should always be the one to go first? & 15 & 20 & 25 & 30 \\
\hline I'll take sugar and cream in my coffee. & 21 & 17 & 28 & 28 \\
\hline Overall Averages & 23 & & 28 & \\
\hline
\end{tabular}

\section{Discussion}

The results of Experiment 2 indicate that although observers are able to extract some lexical information from visual-only displays of speech, the quantity of available lexical information is limited. A higher percentage of lexical items was perceived in those stimuli that were common phrases. At most, the participants were able to recognize $57 \%$ of the lexical items present in certain stimuli; however, on most trials, accuracy was closer to $25 \%$. This finding is consistent with the results of previous studies in which it was reported that normal-hearing observers are typically only able to identify between $20 \%$ and $50 \%$ of lexical items from visual-only displays of speech (e.g., Bernstein et al., 1998; Demorest \& Bernstein, 1992). That observers do poorly when attempting to retrieve lexical information from visual-only speech suggests that the high sensitivity to language differences may be the result of additional information available in the visual signal. Converging evidence that lexical information is not the only available information in the visual signal comes from a lexical-decision experiment (Ronquest, Levi, \& Pisoni, 2007) with the Spanish and English words used in Experiments $3 \mathrm{~A}$ and $3 \mathrm{~B}$ below. In the lexical-decision experiment, observers were presented with visual-only signals of both English and Spanish words and were asked to decide whether a stimulus was a word or a nonword. The experiment revealed that many of the Spanish stimuli were classified as words (52\% for Spanish, $60 \%$ for English). This result suggests that basing language identification on whether a stimulus is recognized as a word would result in much lower sensitivity scores. This previous study further supports the hypothesis that lexical information alone cannot account for the high levels of accuracy on the language-identification task. One possible source of information that may supplement lexical information as a cue to language identity is the rhythmic difference between English and Spanish.

\section{EXPERIMENTS 3A AND 3B Rhythmic Cues to Language Identification}

Experiments $3 \mathrm{~A}$ and $3 \mathrm{~B}$ were designed to assess the contribution of rhythmic differences to visual-only language identification. To eliminate lexical information, the visual stimuli were temporally reversed (hereafter, backward stimuli). Thus, in the backward stimuli (e.g., breakfast is ready, [brekfəstızreri]), the visemes associated with each segment would be reversed, generating strings of nonsense visemes. ${ }^{1}$ Although it is true that temporal reversal preserves coarse-grained segmental information (e.g., full labial closure for a biliabial stop), the phonotactic legality of many sequences will not be retained (e.g., [fæst] is legal but [tsæf ] is not). However, because the visemes associated with vowels and consonants are retained in the backward stimuli and because temporal reversal does not alter the duration of these visemes, the temporal/rhythmic differences between Spanish and English should be relatively well maintained under this manipulation. Furthermore, previous studies on visualonly speech perception have reported that observers are able to extract temporally dependent information such as speaking-rate and stress differences from visual-only displays of speech (Bernstein, Eberhardt, \& Demorest, 1986; Green, 1987). Thus, backward versions of the visual-only stimuli were included in the stimulus set to assess whether the participants could make accurate judgments about the language when lexical information was unavailable.

As was discussed earlier, Spanish is a syllable-timed language, and English is a stress-timed language. Thus, in Spanish, the durations of vocalic gestures are more regular, 
whereas in English they are more variable. Temporal reversal of sentences preserves these timing differences, but eliminates fine-grained gestural-articulatory information and temporal order of visemes necessary for lexical access. If the participants can use differences in linguistic rhythm to identify languages, they should be able to identify languages in the temporally reversed stimuli, although they should be more accurate in the forward condition where both lexical and rhythmic information are present. In contrast, if the participants are unable to use rhythmic cues, performance on the backward stimuli should be extremely poor.

In Experiments 3A and 3B, we also examined the effect of stimulus length, which has been previously shown to improve language discrimination in visual-only speech (Soto-Faraco et al., 2007). Longer utterances provide larger samples of speech and, thus, more opportunity for the observer to extract information necessary for accurate language identification. For this reason, both sentences and isolated words were used in Experiments 3A and 3B to test whether longer utterances would facilitate language identification. We were also interested in determining whether the limited information from isolated words would provide sufficient information to permit reliable language identification.

\section{Experiment 3A: Naive Observers}

\section{Method}

Stimulus materials. Three hundred twenty video clips were used in Experiment 3A: 20 English and 20 Spanish sentences, and 20 English and 20 Spanish words, each spoken by two talkers and presented in two directions (forward and backward). The 80 forward sentences were the same as those used in Experiment 1. The 80 isolated word stimuli were recorded under the same conditions as were the sentences described in Experiment 1. The words included the days of the week, the names of animals, and the words for the numbers $1,3-5$, and 7-10 (see Appendix B). All video clips were temporally reversed on an Apple Macintosh computer using Final Cut Pro.

To confirm that the Spanish and English stimuli used in this experiment were produced with the expected rhythmic differences, we conducted acoustic analyses of the sentence-length utterances using the normalized pairwise variability index (nPVI). The analyses were not conducted for the isolated word stimuli, because many of these stimuli were monosyllabic, making it impossible to establish the variability of consecutive vowels. The vocalic nPVI was computed for each of the 80 sentences. To compute the vocalic nPVI, the duration of each vowel in a particular sentence was measured, and these consecutive vowel durations were put into Equation 1 from Grabe and Low (2002), yielding a vocalic nPVI for each sentence. Here, $d_{k}$ refers to the duration of the $k$ th vowel, and $m$ refers to the total number of vowels in the sentence.

$$
\mathrm{nPVI}=100 \cdot \sum_{k=1}^{m-1}\left|\frac{d_{k}-d_{k+1}}{\left(d_{k}+d_{k+1}\right) / 2}\right| /(m-1)
$$

The nPVI provides a measure of the variability of vowel durations in an utterance. Vowel durations that differ greatly between adjacent syllables yield large values, whereas vowels that are nearly equal in duration yield smaller values. Larger nPVIs are typical of stresstimed languages like English, in which vowels tend to be reduced in unstressed syllables; small nPVIs are found in syllable-timed languages, in which vowel durations are relatively stable across stressed and unstressed syllables.

The average vocalic nPVI values for each of the two talkers' English and Spanish sentences are displayed in Table 3. Separate one-
Table 3

Means and Standard Deviations for Vocalic Normalized Pairwise Variability Index Measures

\begin{tabular}{lccccc}
\hline & \multicolumn{2}{c}{ English } & & \multicolumn{2}{c}{ Spanish } \\
\cline { 2 - 3 } \cline { 5 - 6 } & $M$ & $S D$ & & $M$ & $S D$ \\
\hline Male talker & 47.2 & 16.9 & & 30.1 & 6.5 \\
Female talker & 52.0 & 16.5 & & 28.9 & 5.6 \\
\hline
\end{tabular}

way ANOVAs were conducted on the male and the female talker to determine whether rhythmic differences existed between the English and Spanish stimuli. A significant difference between the two languages was found for both the female talker $[F(1,39)=34.91, p<$ $.001]$ and the male talker $[F(1,39)=17.77, p<.001]$, indicating that both talkers produced different rhythmic patterns when speaking English and Spanish. Specifically, as was anticipated, English stimuli were produced with higher variability of vowel duration (higher nPVI), whereas Spanish stimuli were produced with less variability of vowel duration (smaller nPVI).

The results of this acoustic analysis confirm that the English and Spanish stimuli used in Experiments 2 and 3 exhibit different rhythmic patterns. As was expected from previous studies, the nPVI measures were significantly higher in English for both the male and the female talker than they were in Spanish, indicating greater variability in the timing of vocalic segments in English. Furthermore, the values found here are similar to those reported in other published studies, with vocalic nPVIs in the mid-50s for English and near 30 for Spanish (Grabe \& Low, 2002). As was discussed in the introduction, vocalic segments correspond to different visemes than do consonants, and the duration of these vocalic visemes is accessible in the visual signal. Thus, the existence of temporal (i.e., rhythmic) differences in the acoustic signal implies the existence of visual rhythmic differences in the stimuli used here. In the present experiment, we examined whether observers can use these visual temporal/ rhythmic differences to aid language identification for languages that belong to different rhythmic classes.

Participants. Thirty-four students who were enrolled in introductory psychology classes at Indiana University participated in Experiment 3A. Two participants were eliminated for not following the instructions. None of the remaining 32 participants who took part in Experiment 3A had completed Experiment 1 or Experiment 2. All of the participants were monolingual speakers of English who reported little or no knowledge of Spanish and no history of speech or hearing disorders at the time of testing. The participants received partial course credit for their participation.

Procedure. The general procedure for Experiment 3A was similar to that used in Experiment 1. The participants were presented with two blocks of 160 stimuli. One block consisted of forward and backward words; the other block consisted of forward and backward sentences. The presentation of the blocks was counterbalanced across participants. After seeing each video clip, the participants were asked to decide whether the person in the video was speaking English or Spanish. A button box was used to record the participants' responses. The participants were not informed that half of the video clips were time reversed. No feedback was provided.

\section{Results}

As in Experiment 1, nonparametric measures of sensitivity $\left(A^{\prime}\right)$ and bias $\left(B_{\mathrm{D}}^{\prime \prime}\right)$ were calculated for each participant. The mean values of $A^{\prime}$ and $B_{\mathrm{D}}^{\prime \prime}$ are presented in Figures 3 and 4. A one-sample $t$ test of $A^{\prime}$ scores for the four conditions revealed that the participants scored statistically above chance in all conditions [forward sentences, $t(31)=14.84, p<.001 ;$ backward sentences, $t(31)=$ $7.11, p<.001$; forward words, $t(31)=10.31, p<.001$; backward words, $t(31)=9.68, p<.001]$, indicating that 


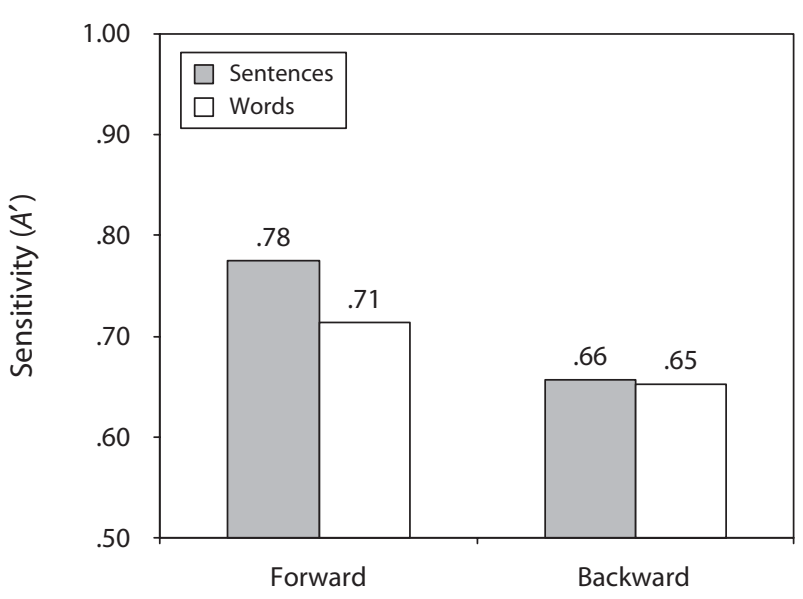

Figure 3. Mean sensitivity $\left(A^{\prime}\right)$ in all four stimulus conditions for Experiment 3A.

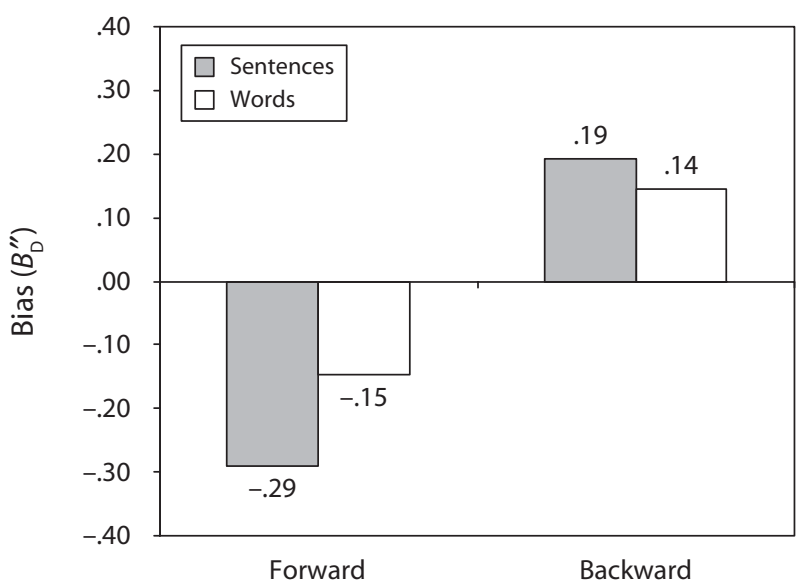

Figure 4. Mean bias $\left(B_{\mathrm{D}}^{\prime \prime}\right)$ in all four stimulus conditions for Experiment $3 \mathrm{~A}$. Negative values indicate a bias to respond English; positive values indicate a bias to respond Spanish.

the participants were able to reliably identify the language from visual-only displays in all conditions. Critically, the languages were accurately identified in the backward condition for both isolated words and sentences.

A repeated measures ANOVA of $A^{\prime}$ scores with stimulus direction (forward vs. backward) and length (words vs. sentences) as within-subjects variables revealed a significant main effect of stimulus direction $[F(1,31)=$ $21.53, p<.001]$. The participants were more sensitive to language differences when the stimuli were presented forward $\left(A^{\prime}=.74\right)$ than when they were presented backward $\left(A^{\prime}=.65\right)$. The main effect of stimulus length approached significance $[F(1,31)=3.32, p=.078]$. The direction $\times$ length interaction also approached significance $[F(1,31)=3.49, p=.071]$. Paired-samples $t$ tests of this interaction revealed that sensitivity was affected by length in the forward condition only. Sensitivity to language differences in the forward condition was greater with sentences than with words $[t(19)=2.57, p=.015]$. In the backward condition, the benefit of stimulus length was not significant. Thus, the direction $\times$ length interaction reduced the effect of stimulus length. Notably, in the forward condition, in which lexical and other fine-grained segmental information was available, the observers performed better when given longer stimuli. In the backward condition, in which the observers could rely on rhythmic differences and in which lexical information was unavailable, the effect of increased length disappeared.

A one-sample $t$ test of bias scores (tested against 0 ) revealed a significant negative (English) bias for the forward sentences $[t(31)=-5.14, p<.001]$ and forward words $[t(31)=-2.10, p=.044]$ and a significant positive (Spanish) bias for backward sentences $[t(31)=3.37, p=.002]$ and backward words $[t(31)=2.52, p=.017]$. A repeated measures ANOVA revealed a significant main effect of direction $[F(1,31)=38.57, p<.001]$, indicating that the participants were more biased to respond English for the forward stimuli $\left(B_{\mathrm{D}}^{\prime \prime}=-.22\right)$ and Spanish to the backward stimuli $\left(B_{\mathrm{D}}^{\prime \prime}=.17\right)$. In this task, the participants judged the unusual (i.e., backward) stimuli as more likely to be Spanish, the unfamiliar language. The main effect of length was not significant, and the direction $\times$ length interaction only approached significance $[F(1,31)=3.15, p=.086]$. Similar to sensitivity, the bias measures indicated a greater effect of stimulus length in the forward condition.

Converging evidence for the use of rhythmic differences in visual-only language identification comes from correlations between the nPVI measures and an item analysis of the percent of English responses to individual stimuli. Separate bivariate correlations were conducted on the forward and backward stimuli to establish the utility of the rhythmic measure on responses. A significant positive correlation was found in both the forward condition $(r=$ $.54, n=80, p<.001)$ and the backward condition $(r=.54, n=80, p<.001)$. These positive correlations suggest that as the nPVI increases - that is, as the stimulus is more English-like with respect to rhythm - the percent of English responses also increases. What is noteworthy here is that the correlation remained high even in the backward condition, in which lexical cues were absent.

\section{Experiment 3B: Informed Observers}

\section{Method}

A modified version of Experiment 3A was conducted to examine the effect of direction when participants were explicitly told that some of the stimuli had been temporally reversed. The combined results of $A^{\prime}$ and $B_{D}^{\prime \prime}$ from Experiment $3 \mathrm{~A}$ suggest that the participants had greater difficulty discriminating between English and Spanish with the unnatural backward sentences. Because the participants were unaware of the manipulation of these utterances, they were more likely to respond Spanish to these unusual stimuli, which did not appear to contain any lexical information. Because temporal reversal of the stimuli eliminated fine articulatory details and lexical cues, we investigated whether the participants' expectations would change response patterns. That is, does awareness of the temporal manipulation affect how observers perform a languageidentification task?

Stimulus materials. The stimulus materials used in Experiment 3B were the same as those used in Experiment 3A.

Participants. A total of 33 introductory psychology students took part in this experiment. Thirteen participants were eliminated because they had studied Spanish $(n=3)$, had native Spanish- 
speaking parents $(n=1)$, or had undergone speech therapy $(n=1)$; because of a computer malfunction $(n=4)$; and to equalize the groups $(n=4)$. The remaining 20 participants met the same criteria as those in Experiment 3A. The participants received partial course credit for their participation. None of the participants in Experiment 3B had taken part in any of the previous experiments.

Procedure. Four blocks of visual-only stimuli (forward words, forward sentences, backward words, and backward sentences) were presented to the participants. Prior to the presentation of each stimulus block, the participants were explicitly told whether the stimuli would be presented forward or backward and whether they would be viewing single words or whole sentences. Blocks were counterbalanced across participants. After viewing each video clip, the participants were asked to decide whether the person in the video was speaking English or Spanish. As in Experiment 3A, each block consisted of an equal number of English and Spanish video clips spoken by both the male talker and the female talker. No feedback was provided.

\section{Results}

The same statistical analyses carried out on the data from Experiment 3A were performed on the data collected in Experiment 3B. A summary of the $A^{\prime}$ scores is shown in Figure 5. A one-sample $t$ test of sensitivity $\left(A^{\prime}\right)$ scores revealed that, as in Experiment $3 \mathrm{~A}$, the participants identified the languages at levels above chance in all four conditions [forward sentences, $t(19)=6.55, p<.001$; backward sentences, $t(19)=6.15, p<.001$; forward words, $t(19)=5.09$, $p<.001$; backward words, $t(19)=3.20, p=.005]$. A repeated measures ANOVA with stimulus direction (forward vs. backward) and length (word vs. sentence) as withinsubjects variables revealed significant main effects of direction $[F(1,19)=5.90, p=.025]$ and length $[F(1,19)=$ $5.27, p=.033]$. As was observed in Experiment 3A, the participants were more sensitive to language differences when the stimuli were presented forward $\left(A^{\prime}=.66\right)$ than when they were presented backward $\left(A^{\prime}=.61\right)$ and were also more accurate with sentences $\left(A^{\prime}=.66\right)$ than words $\left(A^{\prime}=.60\right)$. The direction $\times$ length interaction was also significant $[F(1,19)=10.07, p=.005]$. Post hoc pairedsamples $t$ tests on this interaction revealed that sensitivity was affected by length in the backward condition $[t(19)=$ $-3.14, p=.005]$. Thus, when the observers were made aware of the manipulation and were explicitly told that the

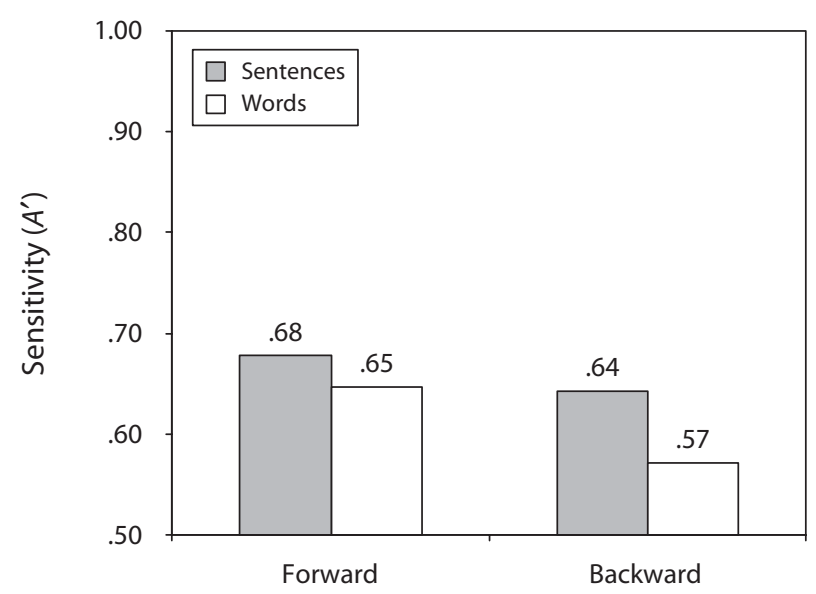

Figure 5. Mean sensitivity $\left(A^{\prime}\right)$ in all four stimulus conditions for Experiment 3B.

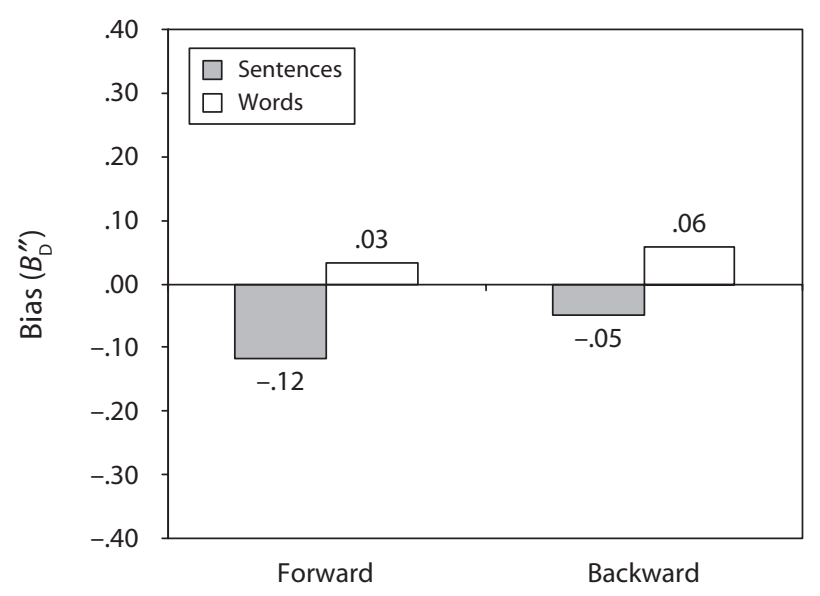

Figure 6. Mean bias $\left(\boldsymbol{B}_{\mathrm{v}}^{\prime \prime}\right)$ in all stimulus conditions for Experiment 3B. Negative values indicate a bias to respond English; positive values indicate a bias to respond Spanish.

stimuli were backward, they exhibited the expected effect of length. In the backward condition, they were aware that lexical information was unavailable, and therefore, they knew that they must rely on other differences between the two languages. Sentence-length utterances provided more reliable rhythmic differences between the two languages because of the existence of more vowels from which to gather comparative measures of temporal variability. In contrast to the results of Experiment 3A, the effect of length was not significant in the forward direction.

Measures of response bias $\left(B_{\mathrm{D}}^{\prime \prime}\right)$ were also calculated. A summary is presented in Figure 6. One-sample $t$ tests on the bias scores revealed that the participants exhibited a significant response bias only for the forward sentences $[t(19)=-2.83, p=.01]$. The response biases for the other conditions did not differ significantly from 0 . Thus, an additional consequence of informing the observers of the directional manipulation was a reduction in response bias. A repeated measures ANOVA with stimulus direction (forward vs. backward) and length (word vs. sentence) as within-subjects variables revealed a significant main effect of direction $[F(1,19)=5.93, p=.025]$. Overall, the observers were more biased to respond English in the forward condition than in the backward condition $\left(B_{\mathrm{D}}^{\prime \prime}=\right.$ -0.09 and 0.10 , respectively). The main effect of stimulus length was also significant $[F(1,19)=4.89, p=.039]$. On average, the participants were more likely to respond $E n$ glish with the longer stimuli. This main effect suggests that when listeners know why stimuli look unusual (i.e., they are backward), they no longer use stimulus naturalness as their metric for responding English versus Spanish. The direction $\times$ length interaction was also not significant.

As with Experiment 3A, we conducted correlations between nPVI and the percentage of English responses. Significant positive correlations were found for both the forward condition $(r=.55, n=80, p<.001)$ and the backward condition $(r=.40, n=80, p<.001)$. This positive correlation suggests that as nPVI increases (i.e., the rhythmic properties of a stimulus become more like those of English), the percentage of English responses also increases. 


\section{Discussion}

In Experiments 3A and 3B, we examined the contribution of rhythmic information to visual-only language identification by eliminating cues to lexical access using temporally reversed stimuli. As was previously mentioned, rhythmic differences between English and Spanish are accessible and are retained in temporally reversed stimuli. The results of Experiments $3 \mathrm{~A}$ and $3 \mathrm{~B}$ demonstrate that observers can identify languages that differ in rhythmic structure from visual-only stimuli, even in the absence of possible lexical or fine-grained segmental information. The participants' ability to identify languages in the backward condition suggests that they can use rhythmic information to help differentiate languages. We conclude that the rhythmic properties of a language are one cue that participants use to determine language identity from visualonly displays of speech. Correlations between nPVI and the percentage of English responses provide converging evidence for this conclusion. As stimuli become more like English, responses of English increase. This is true even in the backward conditions, in which the observers did not have access to lexical information, supporting the idea that rhythmic differences in the stimuli contribute to language identification in visual-only speech perception.

Not surprisingly, sensitivity to the language differences was greater when the stimuli were presented in the forward direction, where all cues to language identity were present. As was found in previous studies, stimulus length affected sensitivity; sentence-length stimuli provided more information (i.e., more chances to recognize the language and more temporal, rhythmic cues) for language identity than did isolated words. The effect of length was not found when naive observers were presented with backward stimuli. For these observers, the unfamiliarity or unnaturalness of the backward stimuli likely caused them to conclude that the language was Spanish. This is corroborated by the bias data in Experiment 3A, in which the observers showed a bias toward Spanish responses in the backward condition. Critically, the observers in both Experiment 3A and Experiment 3B were sensitive to language differences in the backward condition, suggesting that temporal, rhythmic information can aid language identification in visual speech.

Informing the participants of the temporal manipulation primarily resulted in a change in response bias. When the participants were unaware of the temporal reversal (Experiment 3A), they were significantly biased toward Spanish responses in the unfamiliar backward condition and toward English responses in the familiar-looking forward condition. In contrast, the biases of the informed observers (Experiment 3B) did not differ from 0 in either of the backward conditions, suggesting that they no longer relied on familiarity or naturalness of the stimuli. Thus, explicitly informing the observers that some blocks of stimuli were played backward effectively altered their response strategy.

The results of Experiments 3A and 3B show that the rhythmic properties of a language, which were maintained in the temporally reversed versions of the stimuli, provided sufficient cues to language identity. Thus, it is not necessary for lexical information to be present for reliable language identification to occur. In the forward condition, where both the rhythmic and lexical properties of the language were present, overall performance was enhanced. Greater sensitivity to the linguistic differences in the forward stimuli suggests that a combination of rhythmic cues and lexical information is more useful in this task than having only one set of cues available.

\section{GENERAL DISCUSSION AND CONCLUSIONS}

In this article, we investigated how observers identify languages from visual-only displays of speech. Our first goal was to replicate and extend the findings of SotoFaraco et al. (2007), using a different experimental paradigm and a different set of languages. Overall, the results of Experiment 1 confirmed the earlier findings that language identification is possible from visual information alone. Although we failed to replicate the effect of linguistic experience (monolingual vs. bilingual) on sensitivity, its effect was observed in response bias; the bilingual speakers exhibited a greater bias toward their native language than did the monolingual speakers. This response bias was the largest with the bilingual English speakers, although a similar trend was found for the Spanish bilinguals, with more bias toward Spanish.

Our second goal in this investigation was to explore what sources of information observers use to identify languages. This was done by examining their reliance on lexical and rhythmic information. In Experiment 2, we assessed the extent to which lexical items could be identified in visual-only speech. The results indicated that although the observers were able to identify some lexical items from a visual-only display, the quantity of available lexical information in this modality is very limited. Common words and phrases were identified more accurately than less common lexical items, but overall, the percentage of correct word identification was low.

Because previous research has shown that prelinguistic infants can differentiate languages belonging to different rhythmic categories (Mehler et al., 1988; Nazzi et al., 1998; Nazzi et al., 2000), we examined another possible cue to language identity: linguistic rhythm. Acoustic analysis of the auditory signals confirmed that our Spanish and English stimuli differed with respect to linguistic rhythm. Because vowel and consonant gestures are distinguishable from visual-only speech, we hypothesized that these rhythmic differences between Spanish and English could be used by observers to aid in language identification. This hypothesis was supported by studies of language discrimination from auditory stimuli, which retained only consonant versus vowel durational cues, eliminating all segmental and pitch information (Ramus \& Mehler, 1999). To simultaneously eliminate lexical information and retain the relative temporal relationships between consonants and vowels (i.e., linguistic rhythm), the visual signal was temporally reversed in Experiments $3 \mathrm{~A}$ and $3 \mathrm{~B}$. Critically, the participants reliably identified the language in the backward condition. This 
finding suggests that language identification is possible in the absence of lexical information. That the observers were able to reliably identify languages in the backward condition suggests that the rhythmic differences between the two languages provide information to support language identification. As was expected, when both rhythmic and lexical information were available (i.e., forward condition), the observers performed significantly better. In Experiments 3A and 3B, we also tested the effect of utterance length. Similar to Soto-Faraco et al.'s (2007) findings, the participants showed better performance with sentence-length than with word-length utterances.

Taken together, the results of these three experiments demonstrate that the visual speech signal contains multiple cues to language identity. In particular, we found that both rhythmic and lexical information are available in the visual signal. By selectively manipulating the type of information available to observers, we were able to examine the contribution of lexical and rhythmic cues separately. Consistent with previous findings reported in the literature (Bernstein et al., 1998; Demorest \& Bernstein, 1992; Lachs, 1999; Lachs et al., 2002), observers can perceive some lexical information from visual speech. Temporal reversal, which retains global timing and segmental (i.e., consonant vs. vowel) information, allowed us to test the contribution of linguistic rhythm. Future investigations of visual-only language identification and discrimination will provide additional insights into how observers carry out these tasks and will assess the extent to which lexical, segmental, and suprasegmental (prosodic and rhythmic) information is accessed during visual-only speech perception. More generally, the results of the experiments presented here provide additional support for the conclusion that speech perception is multimodal in nature, that the visual signal - as well as the auditory signal - is robust and rich with information. Moreover, observers are able to extract and utilize different components of the visual signal in order to make highly detailed judgments about the source and contents of the signal.

\section{AUTHOR NOTE}

This work was supported by grants from the National Institutes of Health to Indiana University (NIH-NIDCD T32 Training Grant DC00012 and NIH-NIDCD Research Grant R01 DC-00111). We thank Luis Hernandez for assistance with programming, and Althea Bauernschmidt and Vidhi Sanghavi for their help in running participants. Special thanks are extended to Manuel Díaz-Campos for his help in collecting data from the monolingual Spanish participants in Venezuela. We also thank three anonymous reviewers and the associate editor for their helpful comments on previous drafts. Correspondence concerning this article should be addressed to R. E. Ronquest, Speech Research Laboratory, Department of Psychological and Brain Sciences, Indiana University, 1101 East Tenth Street, Bloomington, IN 47405-1301 (rronques@indiana.edu).

\section{REFERENCES}

Bernstein, L. E., Demorest, M. E., \& Tucker, P. E. (1998). What makes a good speechreader? First you have to find one. In R. Campbell, B. Dodd, \& D. Burnham (Eds.), Hearing by eye II: Advances in the psychology of speechreading and auditory-visual speech (pp. 211227). East Sussex, U.K.: Psychology Press.

Bernstein, L. E., Eberhardt, S. P., \& Demorest, M. E. (1986). Judgments of intonation and contrastive stress during lipreading. Journal of the Acoustical Society of America, 80(Suppl. 1), S78.
Campbell, C. S., \& Massaro, D. W. (1997). Perception of visible speech: Influence of spatial quantization. Perception, 26, 627-644.

CAmpbell, R., \& DodD, B. (1980). Hearing by eye. Quarterly Journal of Experimental Psychology, 32, 85-99.

Davis, H., \& Silverman, S. R. (EDs.) (1970). Hearing and deafness (3rd ed.). New York: Holt, Rinehart \& Winston.

Demorest, M. E., \& Bernstein, L. E. (1992). Sources of variability in speechreading sentences: A generalizability analysis. Journal of Speech \& Hearing Research, 35, 876-891.

Donaldson, W. (1992). Measuring recognition memory. Journal of Experimental Psychology: General, 121, 275-277.

Grabe, E., \& Low, E. L. (2002). Durational variability in speech and the rhythm class hypothesis. In C. Gussenhoven \& N. Warner (Eds.), Papers in laboratory phonology (Vol. 7, pp. 515-546). Berlin: Mouton de Gruyter.

GreEN, K. P. (1987). The perception of speaking rate using visual information from a talker's face. Perception \& Psychophysics, 42, 587-593.

GrIER, J. B. (1971). Nonparametric indexes for sensitivity and bias: Computing formulas. Psychological Bulletin, 75, 424-429.

Hamilton, R. H., Shenton, J. T., \& Coslett, H. B. (2006). An acquired deficit of audiovisual speech processing. Brain \& Language, 98, 66-73.

HARDISON, D. M. (2003). Acquisition of second language speech: Effects of visual cues, context, and talker variability. Applied Psycholinguistics, 24, 495-522.

Hazan, V., Sennema, A., \& Faulkner, A. (2002). Audiovisual perception in L2 learners. In J. H. L. Hansen \& B. Pellom (Eds.), Proceedings of the 7th International Conference on Spoken Language Processing (pp. 1685-1688). Denver.

Huarte, A., Molina, M., Manrique, M., Olleta, I., \& GarcíaTAPIA, R. (1996). Protocolo para la valoración de la audición y el lenguaje, en lengua española, en un programa de implantes cocleares. Acta Otorrinolaringologica Española, 47(Suppl. 1), 1-14.

Kaiser, A. R., KirK, K. I., Lachs, L., \& Pisoni, D. B. (2003). Talker and lexical effects on audiovisual word recognition by adults with cochlear implants. Journal of Speech, Language, \& Hearing Research, 46, 390-404

LACHS, L. (1999). Use of partial stimulus information in spoken word recognition without auditory stimulation. In Research on Spoken Language Processing (Progress Rep. No. 23). Bloomington: Indiana University, Department of Psychology, Speech Research Laboratory.

LaChs, L., WeIss, J. W., \& Pisoni, D. B. (2002). Use of partial stimulus information by cochlear implant patients and normal hearing listeners in identifying spoken words: Some preliminary analyses. Volta Review, 102, 303-320.

Massaro, D. W. (1987). Speech perception by ear and eye: A paradigm for psychological inquiry. Hillsdale, NJ: Erlbaum.

McGurK, H., \& MACDonALD, J. (1976). Hearing lips and seeing voices. Nature, 264, 746-748.

Mehler, J., JusczyK, P., Lambertz, G., Halsted, N., Bertoncini, J., \& Amiel-Tison, C. (1988). A precursor of language acquisition in young infants. Cognition, 29, 143-178.

Montgomery, A. A., \& JACKSON, P. L. (1983). Physical characteristics of the lips underlying vowel lipreading performance. Journal of the Acoustical Society of America, 73, 2134-2144.

Navarra, J., \& Soto-Faraco, S. (2007). Hearing lips in a second language: Visual articulatory information enables the perception of second language sounds. Psychological Research, 71, 4-12.

Nazzi, T., Bertoncini, J., \& Mehler, J. (1998). Language discrimination by newborns: Toward an understanding of the role of rhythm. Journal of Experimental Psychology: Human Perception \& Performance, 24, 756-766.

NAZZI, T., JusczYK, P. W., \& Johnson, E. K. (2000). Language discrimination by English-learning 5-month-olds: Effects of rhythm and familiarity. Journal of Memory \& Language, 43, 1-19.

PIKE, K. (1946). The intonation of American English (2nd ed.). Ann Arbor: University of Michigan Press.

Ramus, F., \& Mehler, J. (1999). Language identification with suprasegmental cues: A study based on speech resynthesis. Journal of the Acoustical Society of America, 105, 512-521.

Ramus, F., Nespor, M., \& Mehler, J. (1999). Correlates of linguistic rhythm in the speech signal. Cognition, 73, 265-292.

Ronquest, R. E., Levi, S. V., \& Pisoni, D. P. (2007). Language identi- 
fication from visual-only speech. Research on Spoken Language Processing (Progress Report No. 28, pp. 95-118). Bloomington: Indiana University, Department of Psychology, Speech Research Laboratory.

Rosenblum, L. D., \& Salda ̃̃A, H. M. (1998). Time-varying information for visual speech perception. In R. Campbell, B. Dodd, \& D. Burnham (Eds.), Hearing by eye II: Advances in the psychology of speechreading and auditory-visual speech (pp. 61-81). Hove, U.K.: Psychology Press.

Soto-Faraco, S., Navarra, J., Weikum, W. M., Vouloumanos, A., Sebastián-Gallés, N., \& Werker, J. F. (2007). Discriminating languages by speech reading. Perception \& Psychophysics, 69, 218-237.

Sumby, W. H., \& Pollack, I. (1954). Visual contribution to speech intelligibility in noise. Journal of the Acoustical Society of America, 26, 212-215.

Summerfield, Q. (1987). Some preliminaries to a comprehensive account of audio-visual speech perception. In B. Dodd \& R. Campbell (Eds.), Hearing by eye: The psychology of lip-reading (pp. 3-51). Hillsdale, NJ: Erlbaum.
Walden, B. E., Prosek, R. A., Montgomery, A. A., Scherr, C. K., \& JoNES, C. J. (1977). Effects of training on the visual recognition of consonants. Journal of Speech \& Hearing Research, 20, 130-145.

Weikum, W. M., Vouloumanos, A., Navarro, J., Soto-Faraco, S., Sebastián-Gallés, N., \& Werker, J. F. (2007). Visual language discrimination in infancy. Science, 316, 1159.

Werker, J. F., Frost, P. E., \& McGurk, H. (1992). La langue et les lèvres: Cross-language influences on bimodal speech perception. $\mathrm{Ca}$ nadian Journal of Psychology, 46, 551-568.

\section{NOTE}

1. Because segments are coarticulated and because gestural transitions into segments are temporally different from those out of segments, the backward stimuli were not identical to a production of the same string of segments but in the reverse order (i.e., [irerzitsəferb]). Despite this difference, the visemes that are accessible in the backward stimuli will be associated with nonsense strings.

\section{List of English and Spanish Sentences Used in Experiments 1 and 3}

\section{English CID Sentences List \#9-10 (Davis \& Silverman, 1970)}

1. Where can I find a place to park?

2. I like those big red apples we always get in the fall.

3 . You'll get fat eating candy.

4. The show's over.

5. Why don't they paint their walls some other color?

6 . What's new?

7. What are you hiding under your coat?

8 . How come I should always be the one to go first?

9. I'll take sugar and cream in my coffee.

10. Wait just a minute!

11. Breakfast is ready.

12. I don't know what's wrong with the car, but it won't start.

13. It sure takes a sharp knife to cut this meat.

14. I haven't read a newspaper since we bought a television set.

15. Weeds are spoiling the yard.

16. Call me a little later!

17. Do you have change for a five-dollar bill?

18. How are you?

19. I'd like some ice cream with my pie.

20. I don't think I'll have any dessert.

\section{Spanish Sentences, Adaptation of CID List \#9-10 (Huarte, Molina, Manrique,}

\section{Olleta, \& García-Tapia, 1996)}

1. El desayuno está preparado en la mesa.

2. Qué le pasará al coche, que no funciona.

3. ¿Crees que el cuchillo cortará bien la carne?

4. No he leído un periódico desde que compré la televisión.

5. Las malas hierbas están estropeando el jardín de mi casa.

6. Llámame si puedes un poco más tarde, por favor.

7. ¿Tienes cambios de mil pesetas en la cartera?

8. ¿Qué tal estás?

9. Me gustaría tomar un poco de helado de chocolate con la tarta.

10. Creo que no tomaré ningún postre.

11. ¿Dónde puedo encontrar un sitio para aparcar?

12. Me gustan las manzanas grandes y rojas que hay en los árboles.

13. Si comes muchos dulces, vas a engordar.

14. La película ha terminado tarde.

15. ¿Por qué no pintas las paredes de otro color?

16. ¿Cuál es la noticia mas importante hoy?

17. ¿Qué escondes debajo del abrigo azul?

18. Espera un minuto en la puerta del cine.

19. Pondré azúcar y leche en mi café.

20. ¿Cómo puedo ser siempre el primero en llegar? 
APPENDIX B

List of English and Spanish Words Used in Experiment 3

\begin{tabular}{ll}
\hline List of Common English Words & List of Common Spanish Words \\
\hline 1. Monday & 1. Lunes \\
2. Wednesday & 2. Miércoles \\
3. Friday & 3. Viernes \\
4. Saturday & 4. Sábado \\
5. Sunday & 5. Domingo \\
6. One & 6. Uno \\
7. Three & 7. Tres \\
8. Four & 8. Cuatro \\
9. Five & 9. Cinco \\
10. Seven & 10. Siete \\
11. Eight & 11. Ocho \\
12. Nine & 12. Nueve \\
13. Ten & 13. Diez \\
14. Bird & 14. Pájaro \\
15. Fish & 15. Pez \\
16. Chicken & 16. Gallina \\
17. Duck & 17. Pato \\
18. Dog & 18. Perro \\
19. Donkey & 19. Burro \\
20. Giraffe & 20. Jirafa \\
\hline
\end{tabular}

(Manuscript received July 10, 2009;

revision accepted for publication April 4, 2010.) 